\title{
Effect of the Surface Structure on the Electrochemical Behaviors of Electrodeposited Nickel
}

\author{
By Koji Hashimoto*
}

\begin{abstract}
Electrachemical behaviors were measured on electrcdeposited nickel with characteristic preferred orientations and the effect of the surface structure on the behaviors was discussed. In the anodic polarization, the electrcdeposited nickel with the (211) orientation showed a lower initial dissolution potential than the deposits with the $(210)$ or $(110)$ orientation. In the cathodic polarization, the hydrogen evolution potential and the reduction current density at a constant potential were higher in the deposits with the (211) orientation than in the deposits with the other two orientations. It was, therefore, probable that the deposits with the (211) orientation was chemically most reactive among the nickel electrodeposits prepared under the present electrodepositing conditions. This was in good agreement with the result of electron microscopic observation by the author, that is, the deposits with the (211) orientation showed higher densities of corrosion trenches and holes than those with the other two orientations when the deposits were immersed into the corrosive solution for a constant period of time. The corrosion-suffered areas were twin boundaries or stacking faults which put in an appearance on the deposit surface, and a few crystal defects of these sorts were observed in the deposits having the (210) or (110) orientation, while many twin boundaries appeared in the electron micrographs of the deposits with the(211)orientation. From the above point of view, it was anticipated that there would be some difference in the density of these defects by the difference of orientations. It may therefore be predicted that the difference in the density of these defects has bearing upon the chemical reactivity of elctrodeposited nickel.
\end{abstract}

(Received December 15, 1965)

\section{Introduction}

In the nickel electrodeposited on the mechanically polished substrate there appears some characteristic preferred orientations when the thickness of the layer exceeds a few $\mu$, independent of the substrate but controlled by the electrodepositing conditions ${ }^{(1)(2)}$. It was made clear by the author ${ }^{(3)}$ that surface structures of the deposits with these orientations were closely related to twinning and angles between twin planes and substrate surfaces. These differences in the surface structures are expected to give rise to the difference in chemical reactivity of the electrodeposited nickel with a different preferred orientation against corrosive environment. It has been published by the author ${ }^{(4)}$ that in the electro-

* The Research Institute for Iron, Steel and Other Metals, Tohoku University, Sendai, Japan.

(1) G. I. Finch and G. H. Sun: Trans. Faraday Soc., 32 (1936), 852.

(2) G. I. Finch and A. L. Williams : Trans. Faraday Soc., 33 (1937), 564.

(3) K. Hashimoto: Trans. JIM, 6 (1965), 166.

(4) K. Hashimoto: Trans. JIM, 4 (1963), 142.

Trans, J I M deposited nickel grown epitaxially on the chemically polished substrate of a copper single crystal, twinning dislocations and twin boundaries are dissolved initially into a corrosive solution and are chemically most reactive. In the electrodeposited nickel with a preferred orientation, twin boundaries and stacking faults have also been reported to be chemically reactive because of the initial dissolution into the corrosive solution ${ }^{(5)}$. In this case, a number of corrosion holes attacked by the corrosive solution is fairly larger in the deposits with the (211) orientation than in those with the $(210)$ or (110) orientation. It is predicted, therefore, that the electrodeposited nickel with the (211) orientation is chemically unstable among three kinds of the deposits with these orientations prepared under the electrodepositing condition used by the author.

The present investigation was carried out to elucidate the relationship between the electrochemical behaviors of the deposits having different orientations and the difference of the surface structures observed by electron

( 5 ) K. Hashimoto : Trans. JIM, 7 (1966), 8.

1966 Vol. 7 
microscopy using replicas ${ }^{(3)}$. Further, investigation was also made on the relationship between the electrochemical behaviors and the density of the corrosion holes attacked by the corrosive solution which were observed in the transmission electron micrographs obtained from thin top layers of the thick deposits ${ }^{(5)}$.

\section{Experimental Procedures}

When electrodeposited on the mechanically polished copper substrate, the nickel deposits showed a characteristic preferred orientation in which (211), (210) or (110) plane of the deposits is parallel to the substrate surface. The electrodepositing conditions and preferred orientations for the electrodeposited nickel are summarized in Table 1. The current density for electrodeposition was

Table 1 Electrolytic conditions and orientations.

\begin{tabular}{c|l|l|l}
\hline \hline Orientation & \multicolumn{1}{|c|}{$(110)$} & \multicolumn{1}{|c|}{$(210)$} & \multicolumn{1}{c}{$(211)$} \\
\hline $\mathrm{NiSO}_{4} \cdot 7 \mathrm{H}_{2} \mathrm{O}$ & $1 \mathrm{M} / \mathrm{L}$ & $0.82 \mathrm{M} / \mathrm{L}$ & $0 \mathrm{M} / \mathrm{L}$ \\
$\mathrm{NiCl}_{2} \cdot 6 \mathrm{H}_{2} \mathrm{O}$ & 0 & 0.18 & 1 \\
$\mathrm{H}_{3} \mathrm{BO}_{3}$ & 0.48 & 0.48 & 0.48 \\
$\mathrm{pH}$ & 4 & 4 & 4 \\
\hline
\end{tabular}

$47 \mathrm{~mA} / \mathrm{cm}^{2}$, and the electrodepositing solutions were not stirred. The electradeposits were $10 \mu$ in thickness. Immediately after the electrodeposition, electrochemical behaviors of the deposits were examined by means of a potentiostat ${ }^{(6),(7)}$ by measuring the anodic and cathodic polarization curves and variations in reduction current with time at a constant cathodic potential. The polarization curves were measured in a $5 \% \mathrm{H}_{2} \mathrm{SO}_{4}$ aqueous solution by means of the potentiodynamic method at a constant potential sweep of $0.125 \mathrm{~V} / \mathrm{min}$ by changing the variable resistor of the potential compensation circuit at a constant rate. A platinum electrode was used as the auxiliary electrode and a saturated calomel electrode as the reference electrode. The electrolytic cell was kept at a constant temperature of $25^{\circ} \pm 0.5^{\circ} \mathrm{C}$ by means of a thermostat. The water used for aqueous solutions was purified by the ion exchange method, with a specific electrical resistivity of $5 \times 10^{6} \Omega / \mathrm{cm}$ upon purification. Oxygen contained in the $5 \% \mathrm{H}_{2} \mathrm{SO}_{4}$ aqueous solution was removed by bubbling oxygen free nitrogen for $30 \mathrm{~min}$ prior to the measurement. The oxygen free nitrogen was prepared by passing twice through an aqueous solution of $37.5 \mathrm{cc} / \mathrm{L}$ of pyrogallol with $225 \mathrm{~g} / \mathrm{L}$ of $\mathrm{KOH}$ and then twice through a $39 \% \mathrm{Ti}\left(\mathrm{SO}_{4}\right)_{2}$ aqueous solution.

\section{Results}

The cathodic polarization curves of the deposits having the (211), (110) and (210) orientations are shown in Fig. 1. During the cathodic polarization the reduction current begins to flow at a potential of $\sim-0.2 \mathrm{~V}$ (S.C.E.)** and

(6) S. Shimodaira, M. Matsuo, H. Sugawara and H. Ebiko: J. Japan Inst. Mtals, 26 (1961), 100.

(7) K. Hashimoto, T. Goto, W. Suëtaka and S. Shimodaira: Trans. JIM, 6 (1965), 107.

** All potentials are relative to the saturated calomel electrode (S.C.E). increases rapidly at -0.34 to $-0.46 \mathrm{~V}$, resulting in evolution of hydrogen. The hydrogen evolution potential at which the cathodic current begins to increase rapidly is higher in the deposits with the (211) orientation than in those with the $(210)$ or $(110)$ orientation. In the latter

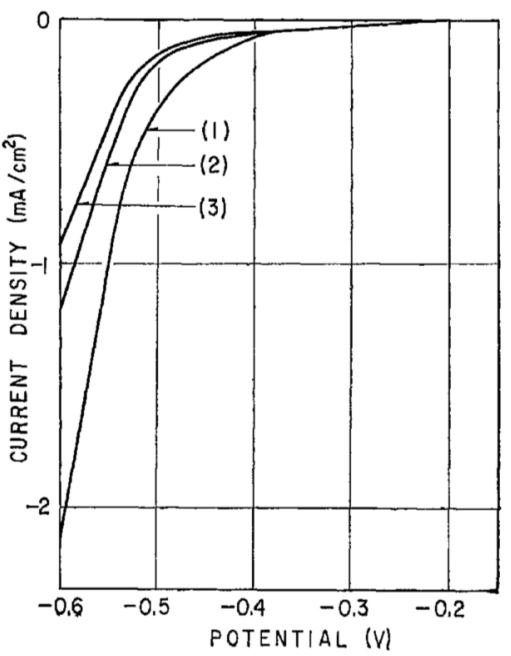

Fig. 1 Cathodic polarization curves.

(1) Electrodeposited nickel with (211) orientation

(2) Electrodeposited nickel with $(110)$ orientation

(3) Electrodeposited nickel with (210) orientetion

two of the deposits, the hydrogen evolution potential of the deposit with the (110) orientation is higher but the difference is not so great. The hydrogen evolution potentials of these deposits are shown in Table 2.

Fig. 2 shows the variation in cathodic current with time during electrolysis on the nickel deposits at a constant potential of $-0.7 \mathrm{~V}$. When kept the cathodic

Table 2 Electrochemical values.

\begin{tabular}{c|c|c|c}
\hline Orientation & $\begin{array}{c}\text { Initial } \\
\text { dissolution } \\
\text { potential (V) }\end{array}$ & $\begin{array}{c}\text { Hydrogen } \\
\text { evolution } \\
\text { potential (V) }\end{array}$ & $\begin{array}{c}\text { Reduction current } \\
\text { density after 30 min } \\
\text { kept at }-0.7 \mathrm{~V} \\
\left(\mathrm{~mA} / \mathrm{cm}^{2}\right)\end{array}$ \\
\hline$(211)$ & -0.03 & -0.37 & 3.84 \\
$(110)$ & 0 & -0.44 & 2.51 \\
$(210)$ & +0.01 & -0.46 & 2.49 \\
\hline
\end{tabular}

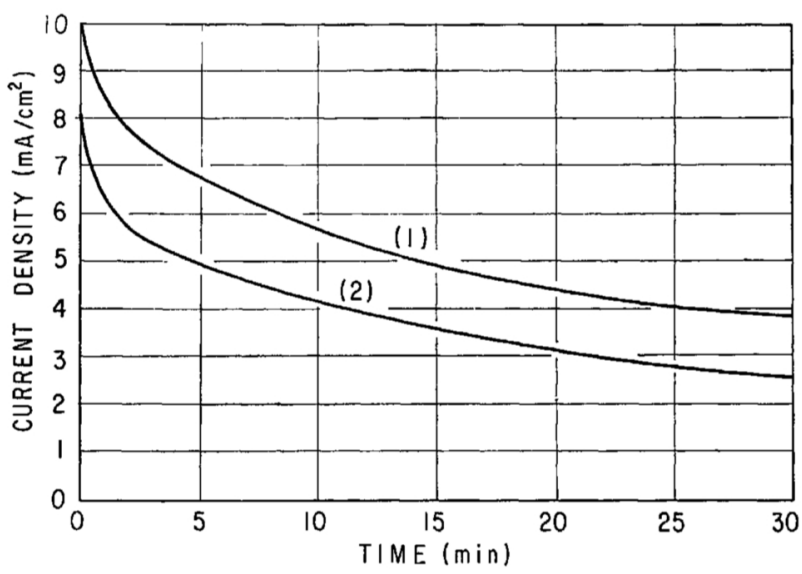

Fig. 2 Cathodic reduction current owing to evolve hydrogen and to reduce surface film at a constant potential of $-0.7 \mathrm{~V}$.

(1) Electrodeposited nickel with (211) orientation.

(2) Electrodeposited nickels with (210) and (110) orientations. 
potential constant, a large cathodic current is initially observed on account of reduction of the film, which exists in the specimen surface, together with evolving hydrogen. The current, however, decreases gradually with time and eventually only the current expended to evolve hydrogen on original surface of electrodeposits is measured. The current of the deposits having the (210) orientation shows nearly the same value as that of the deposits with the (110) orientation so that both curves cannot be separated on the figure. A high cathodic reduction current appears in the deposits with the (211) orientation. In Table 2 are shown the cathodic reduction current observed after holding for $30 \mathrm{~min}$ in electrolysis at the constant potential of $-0.7 \mathrm{~V}$. Fig. 3 shows the anodic

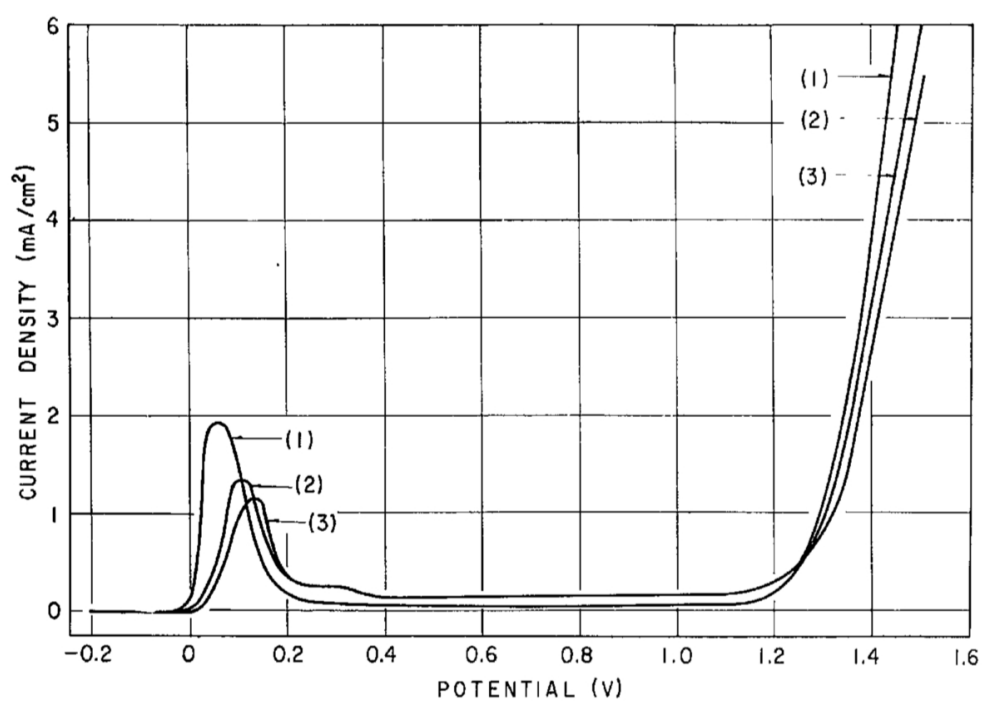

Fig. 3 Anodic polarization curves.

(1) Electrodeposited nickel with (211) orientation.

(2) Electrodeposited nickel with (110) orientation.

(3) Electrodeposited nickel with (210) orientation.

polarization curves of the nickel deposits. Dissolution of the deposits begins at a potential of zero $\mathrm{V}$, and after passing through a maximum and a shoulder of the anodic current, passivation occurs. With further increase of the potential, the anodic current increases rapidly at $\sim$ $+1.2 \mathrm{~V}$ and the evolution of oxygen starts. The initial dissolution potential, at which the dissolution of the nickel deposits begins, is the lowest in the deposits with the (211) orientation and the highest in those with the (210) orientation. These initial dissolution potentials are indicated in Table 2.

The electrochemical behaviors of the deposits with the (211) orientation are different from the deposits having the $(210)$ or (110) orientation, whereas the difference of the behaviors is not clear between the latter two. The above characteristics are not detected during the electrolysis using the solution containing oxygen.

\section{Discussion}

Comparing the deposits having the (211) orientation with those having other two orientations, the initial dissolution potential in the anodic polarization is low, and in the cathodic polarization the hydrogen evolution potential and the cathodic reduction current at a constant po- tential are high. In consequence the deposits with the (211) orientation are chemically most reactive in the deposits prepared under the present electrodepositing conditions.

The author ${ }^{(3)}$ already made clear by means of reflection electron diffraction and electron microscopy using replicas that the surface structure of the electrodeposited nickel is different from each other according to the orientation. Photos. $1 \sim 3$ show the surface structures of the deposits having $(211),(210)$ and (110) orientations. The surface of the deposits with the (211) orientation is covered by hillock-shaped crystals as shown in Photo. 1 . The hillocks were proved to be twins bounded by the (I11) plane parpendicular to the (211) orientation plane and the substrate surface. Owing to the twinning, each hillock shows a characteristic shape which is nearly symmetrical on both sides of the (I11) boundary. Whereas, in the deposits with the (210) orientation (111) planes are oblique to the substrate surface. Twinning in the (210) orientation does not form steep hillocks as in case of the (211) orientation, but many semispherical crystals are grown on the surface of the deposits as seen in Photo. 2. In a reflection election diffraction pattern of the deposits with (110) orientation twin spots of crystals twinned on the $\{111\}$ planes oblique to the (110) plane was not observed. Two $\{111\}$ planes perpendicular to the substrate surface exist in the deposits with the (110) orientation. However, in the deposits prepared under the present electrodepositing condition, twinnings on the two $\{111\}$ planes are rarely observed and the surface of the deposits is smooth as shown in Photo. 3.

Transmission electron microscopy was already applied to the thin surface film of the thick deposits with

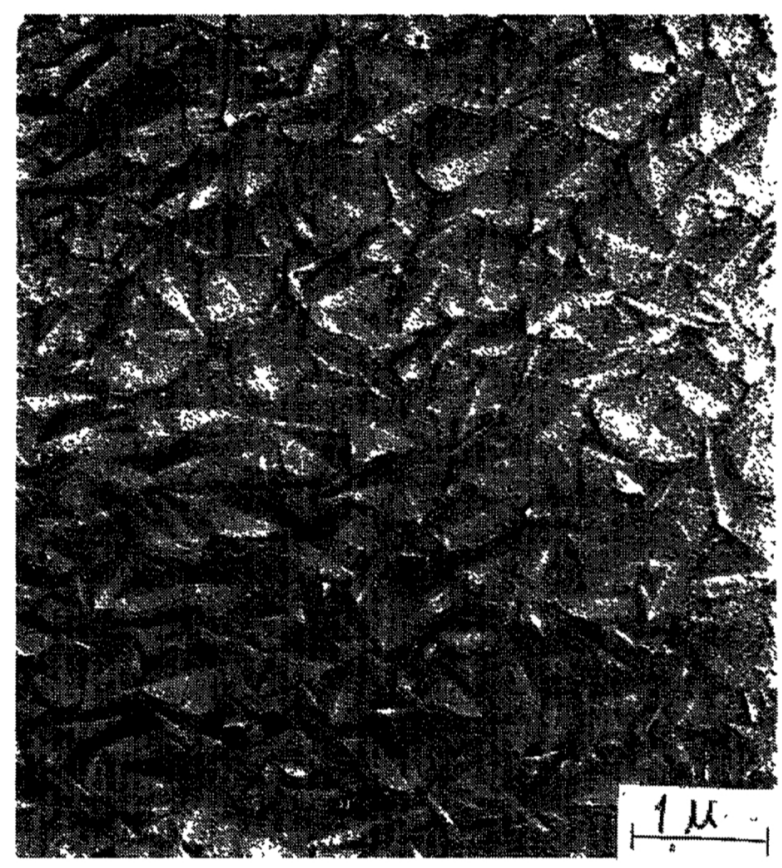

Photo. 1 Surface structure of electrodeposited nickel with (211) orientation ${ }^{(3)}$. 
these preferred orientation ${ }^{(5)}$. Numbers of twin boundaries and stacking faults observable in the electron micrographs were extremely small in the deposits with the $(210)$ or $(110)$ orientation as compared with the (211) orientation.
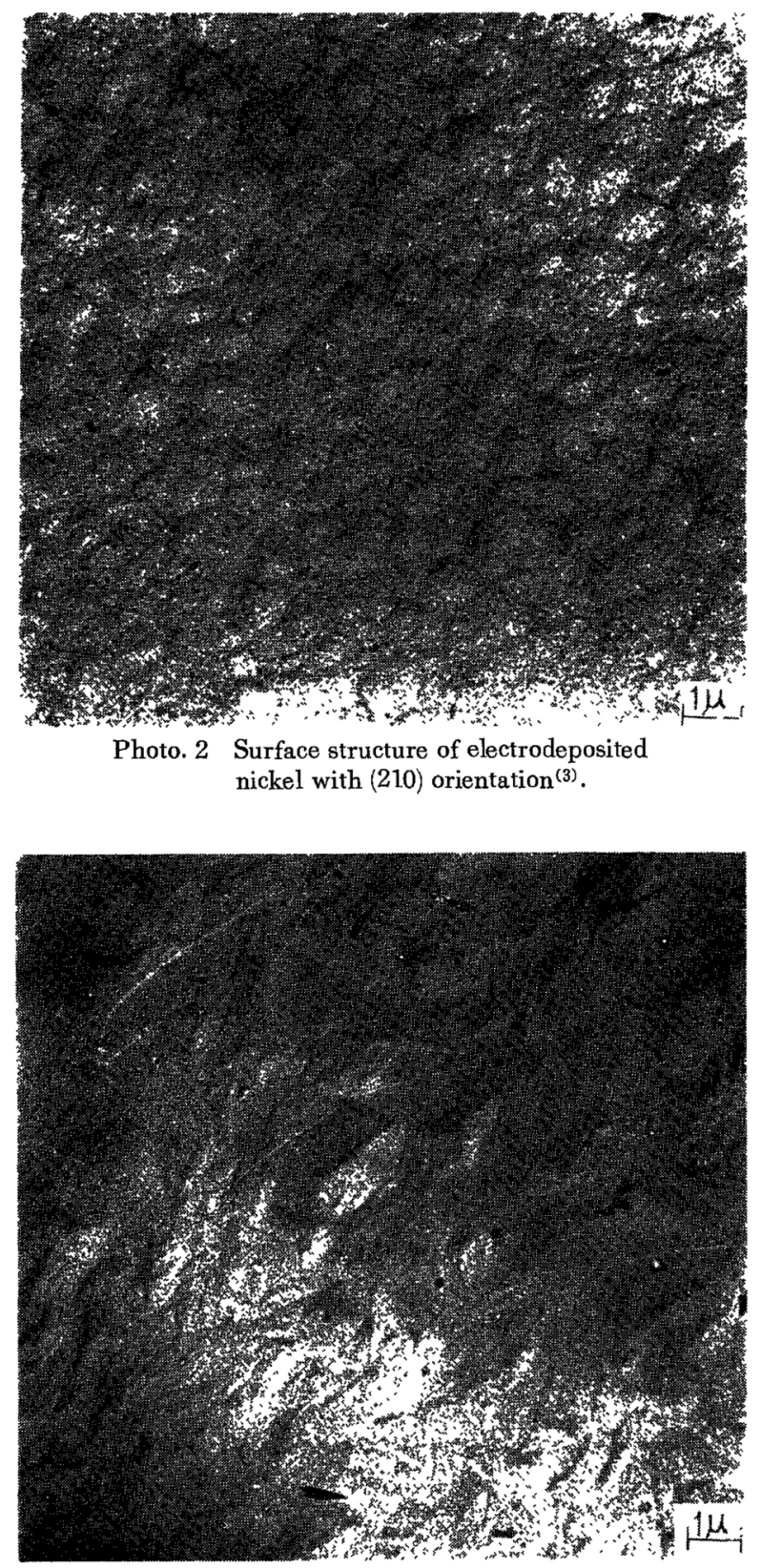

Photo. 3 Surface structure of electrodeposited nickel with $(110)$ orientation $^{(3)}$.

When immersed into the corrosive solution, many corrosion trenches were formed in the deposits with the (211) orientation as shown in Photo. 4, owing to the dissolution of coherent twin boundaries, whereas in the deposit with the (210) or (110) orientation corrosion holes were extremely small in number. It was, therefore, assumed that the density of chemically reactive crystal defects such as twin boundaries and stacking faults varies according to the orientation, and that the deposits with the $(210)$ or $(110)$ orientation are chemically more stable than that with the (211) orientation.

The prediction about the chemical reactivity is in good agreement with the results of electrochemical behaviors measured in the present experiment, and the electrodeposited nickel with the (211) orientation is more chemically reactive than those with other orientations. It is, therefore, probable that the density of the crystal defects observable in the electron micrographs is related

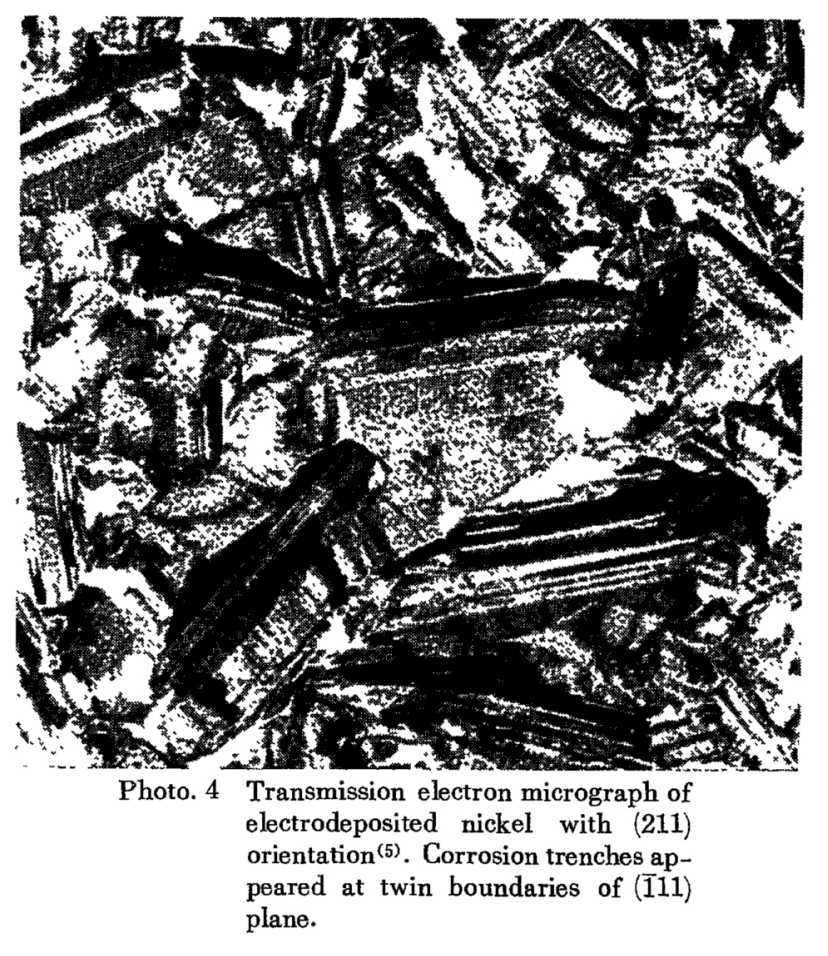

to the chemical reactivity of the electrodeposited nickel, and that the deposits with the (211) orientation is chemically unstable because of a large number of crystal defects on the deposits surface.

\section{Conclusion}

Electrochemical behaviors were measured on the deposits with (211), (210) and (110) orientations. The deposits with the (211) orientation among these deposits showed a low initial dissolution potential in the anodic polarization, and a high hydrogen evolution potential in the cathodic polarization. The cathodic reduction current which was measured at a constant potential was large in the deposits with this orientation. The deposits with the (211) orientation were found to be chemically most reactive among the deposits prepared under the present electrodepositing conditions.

According to the electron microscopic observation reported previously, twin boundaries and stacking faults, which put in an appearance on the deposit surface, were initially attacked by the corrosive solution. Large numbers of these crystal defects and corrosion trenches or holes were observed in the deposits with the (211) orientation. Consequently, the electrochemical behaviors of the electrodeposited nickel seem to be profoundly affected by the density of the chemically reactive crystal defects such as twin boundaries and stacking faults which put in an appearance on the deposit surface. It is likely that corrosion resistivity of electrodeposited metals against corrosive atmospheres can be estimated effectively by investigating the surface structures of the deposits 
by means of reflection electron diffraction and electron microscopy using replicas.

Acknowledgements
The author wishes to express his deep appreciation to Professor Saburo Shimodaira of the Research Institut $\epsilon$ for Iron, Steel and Other Metals, Tohoku University for his valuable discussion during this work. 